PPPL- 2552

DE89 001174

\title{
NONLINEAR GYROKINETIC MAXWELL-VLASOV EQUATIONS USING MAGNETIC COORDINATES
}

\author{
A. BRIZARD \\ Plasma Physics Laboratory, Princeton University \\ Princeton, New Jersey 08543
}

\begin{abstract}
A gyrokinetic formalism using magnetic coordinates is used to derive self-consistent, nonlinear Maxwell-Vlasov equations that are suitable for particle simulation studies of finite- $\beta$ tokamak microturbulence and its associated anomalous transport. The use of magnetic coordinates is an important feature of this work as it introduces the toroidal geometry naturally into our gyrokinetic formalism. The gyrolinetic formalism itself is based on the use of the Action-variational Lie ferturbation method of Cary and Littlejohn, and preserves the Hamiltorian structure of the original Maxwell-Vlasov syatem. Previous nonlinear gyrokinetic set s of equations suitable for particle simulation analysis have considered either electrostatic and shear-Alfvén perturbations in slab geometry, or electrostatic perturbations in soroidal geometry. In this present wc-k, fully electromagnetic perturbations in toroidal geometry are considered.
\end{abstract}

\section{DISCLAIMER}

\begin{abstract}
This report was prepared as an account of work sponsored by an agency of the United States Covernment. Neither the United States Government nor any agency thereof, nor any of their employves, makes any warranty, express or implied, or assumes any legal liability or respois. bility for the accuracy, completeness, or usefulness of any information, apparatus, product, or process disclosed, or represents that its use would not infringe privately owned rights. Reference herein to any specific commercial product, process, or service by trade name, trademark, manufacturer, or otherwise does not necessarily constitute or imply its endorsement, recommendation, or favoring by the United States Government or any agency thereof. The views and opinions of authors expressed herein do not necessarily state or reflect those of the United States Government or any agency thereol.
\end{abstract}




\section{INTRODUCTION}

Particle simulation techniques based on nonlinear gyrokinetic equations have been recently developed by Lee, ${ }^{1,2}$ and have proved to be quite useful for studying various aspects of drift-wave turbulence and its associated anomalous transport. ${ }^{3,4}$

There are three different methods used to derive such nonlinear equations. The first method, referred to as the Standard method, is exemplified by the work of Frieman and Chen ${ }^{5}$ (fully electronagnetic perturbations in general magnetic geometry). It consists of explicitly gyro-averaging the Vlasov equation expressed in lowest-order guiding-center coordinates and separating equilibrium and perturbed parts of the guiding-center distribution function. One obtains a nonlinear gyrokinetic equation for the nonadiabatic part of the perturbed distribution function. Unfortunately, this method involves rather complex algebraic manipulations, fails to provide a clear algorithm indicating how to proceed to higher order, and provides equations that are not directly suitable for particle simulation analysis. It nonetheless provides a basis upon which other methods can be judged.

The second (Hamiltonian) and third (Action-variational) methods require the use of Lie perturbation techniques, particularly those developed by Littlejohn in his work on guidingcenter theory. ${ }^{-s}$ To obtain guiding-center equations of motion, one derives a guiding-center Hamiltonian which has no gyro-angle dependence to any desired order. This provides us with a transformation from physical-space coordinates $(x, v)$ to gujding-center coordinates $(\mathbf{X}, U, \mu, \zeta)$, where $\mathbf{X}$ is the guiding-center position, $U$ is the parallel velocity, $\mu$ is the adiabatic invariant (to asy desired order) known as the magnetic moment, and $\zeta$ is the syro-angle.

The Hamiltonian lie perturbarion method involves the same procedure as the one outlined above. Under perturbation, the guidirg-center Hamiltonian acquires new gyro-angle dependence and, consequently, $\mu$ is no longer invariant. We obtain gyro-center equations of motion by deriving a gyro-center Hamiltonian which has no gyro-angle dependence to any desired order. This provides us with a transformation from guiding-center coordinates $(\mathbf{X}, U, \mu, \zeta)$ to gyro-center coordinates $(\overline{\mathrm{X}}, \bar{U}, \bar{\mu}, \bar{\zeta})$, where now $\bar{\mu}$ is the adjabatic invariant (to any desired order). This method is exemplified by the works of Dubin et al. ${ }^{\circ}$ (electrostatic perturbation in slab geometry), Yang and $\mathrm{Choi}^{10}$ and Hagan and Frieman ${ }^{11}$ (electrostatic perturbations in general geometry), and Hahm et al. ${ }^{12}$ (electrostatic and shear-Alfvén perturbations in slab geometry). A discussion of the works contained in Refs. 5 and 9 to 11 is contained in Hahm et al. ${ }^{12}$

The Action-variational Lie perturbation method represents a generalized form of the Hamiltonian method, as it allows for more general transformations from guiding-center phase space to gyro-center phase space. It recognizes the fact that the Lagrange and Poisson brackets and the Hamiltonian can be perturbed simultaneously. We again obtain gyro-center equations of motion by removing the gyro-angle dependence from the Poisson brackets and the Hamiltonian. New gyro-center coordinates are also produced. This 
method was developed by Littlejohn ${ }^{13}$ and Cary and Littlejohn ${ }^{14}$ and is exemplified by the work of $\mathrm{Hahm}^{15}$ (electrostatic perturbation in general geometry).

Our purpose in the present work is to use the Action-variational Lie perturbation method to derive a set of nonlinear gyrokinetic Maxwell-Vlasov equations, which will describe fully electromagnetic perturbations in toroidal geometry. The ur.perturbed system is represented by an equilibrium tokamak configuration where the plasma pressure and current profiles and magnetic field are related through the (equilibrium) pressure balance equation. The use of magnetic coordinates will enable us to obtain a simple description for the drift motion of gyro-centers, even in complex geometries such as the tokamak configuration.

Two small parameters readily appear in our analysis. The first parameter, $\epsilon \ll 1$, is the ratio of the ion gyroradius to the length scale associated with the equilibrium density nonuniformity and is used to define the so-called nonlinear gyrokinetic ordering, as introduced by Frieman and Chen. ${ }^{5}$ The second parameter, $\epsilon_{B} \ll 1$, is the ratio of the ion gyroradius to the length scale associated with the equilibrium magnetic nonuniformity and is used to define the tolamak ordering. Using a small inverse-aspect-ratio $\left(\epsilon_{0} \ll 1\right)$ tokamak ordering, we have $\epsilon_{B}=\epsilon \epsilon_{0} \ll \epsilon$. We will derive nonlinear equations which contain terms up to order $\epsilon^{2}$ and order $\epsilon_{B}$, i.e., when we consider electromagnetic perturbations, . we will treat the tokamak configuration as uniform (on the ion gyroradius length scale).

Finally, once the transformations from physical-space to guiding-center coordinates and guiding-center to gyro-center coordinates have been derived, it is possible to express the density integrals contained in the set of Maxwell's equations in terms of the total gyrocenter distribution function and gyro-center coordinates. This procedure was used by Lee ${ }^{1}$ and Dubin et al." (Poisson's equation in slab geometry), Yang and Choi ${ }^{10}$ and Frhm ${ }^{15}$ (Poisson's equation in general geometry), and Hahm et al. $^{12}$ (Poisbon and parallel Am,jere equations in slab geometry). We generalize this procedure to the full set of Maxwell's equations in general geometry. Using the same procedure, we also provide an energy integral, useful for numerical simulation purposes. This then completes the derivation of self-consistent, energy-conserving, nonlinear gyrokinetic Maxwell-Vlasov equations.

The remainder of this paper is organized as follows. In section II we describe briefly the Action-variational Lie perturbation method and use it to develop our gyrokinetic formalism. In section III we derive the gyrokinetic Hamiltonian and the gyrokinetic Vlasov equation. In section IV we use our gyrokinetic formaljsm to derive the gyrokinetic Maxwell's equations and the expression for the gyrokinetic energy integral invariant. We present our conclusions in section $\mathrm{V}$ and discuss possible applications of our work. In appendix $A$ we present some notation and terminology used in the theory of Lie transformation of one-forms and density integrals. In Appendix B we present a modified version of Littlejohn's work on guidingcenter theory. In Appendix $\mathrm{C}$ we present the gyrokinetic and tokamak orderings used in this work. Finally, in Appendix D we give the expressions for the Poisson brackets and for 
the unperturbed equations of motion using magnetic coordinates.

\section{LIE PERTURBATION THEORY}

\section{A. Action-variational Lie perturbation method}

In his discussion of various aspects of noncanonical Hamiltonian perturbation theory, Littlejohn ${ }^{13}$ pointed out that the action integral $S \doteq \int L d t$, where $L$ is the Lagrangian function, provided a more general basis to perform Hamiltonian perturbation analysis. More specifically, considering the Poincaré-Cartan fundamental one-form

$$
\gamma \doteq L d t=\mathbf{p} \cdot d \mathbf{q}-H d t
$$

(where the latter expression is given in terms of the familiar canonical variables), one realizes'that the set of canonical transformations of $\mathbf{H}$ (leaving $\mathbf{p} \cdot d \mathbf{q}$ unchanged) can be included in the more general set of noncanonical transformations of the one-form $\gamma$.

As an example, let us consider the problem of charged-particle motion in a uniform magnetic field. In terms of the noncanonical variables $(x, v)$, the one-form $\gamma$ becomes

$$
\gamma=(\mathrm{A}+\mathrm{v}) \cdot d \mathrm{x}-H_{0} d t
$$

where $H_{0}=\frac{1}{2} v^{2}$. We refer to this one-form as the phase-space Lagrangian. Considering perturbations of the form $\mathbf{A} \rightarrow \mathbf{A}+\delta \mathbf{A}$, and $H_{0} \rightarrow H_{0}+\delta \phi$, we notice that both the Hamiltonian and symplectic structure (p-dq part of $\gamma$ ) are perturbed. In addition, magnetic perturbations ( $\delta A)$ appear explicitly in the expression for the perturbed oni-form $\delta \gamma$, a distinct advantage of this method over the Hamiltonian Lie perturbation method.

In the problem of obtaining gyro-center equations of motion, one performs a sequence of transformations $\left\{\cdots T_{2}^{*} T_{1}^{*}\right\}$, where the $n^{\text {th }}$ transformation $T_{n}^{*}$ ( $\epsilon$ denotes the strength of the perturbation) removes the gyro-angle dependence in the $\pi^{\text {th }}$ order terms of the symplectic structure and Hamiltonian. We refer the reader to Appendix A for further details on the theory of transformation of one-forms, and to Refs. 13 and 14 for complete details on the Action-variational Lie perturbation method.

\section{B. Phase-space Lagrangian}

We begin our perturbation analysis by transforming from physical phase space to guiding-center phase space (we use the units in which $e=m=c=1$ ). The unperturbed gyro-averaged phase-space Lagrangian was first derived by Littlejohn. ${ }^{7}$ A modified version of his work produces the expression

$$
\gamma=\left[\mathbf{A}+\rho_{\|} \mathbf{B}-\mu\left(\mathbf{R}+\frac{1}{2}(\hat{b} \cdot \nabla \times \hat{b}) \hat{b}\right)\right] \cdot d \mathbf{X}+\mu d \zeta-\left(\frac{1}{2} \rho_{\|}^{2} B^{2}+\mu B\right) d t,
$$

where $\left(\mathrm{X}, \rho_{\|}, \mu, \zeta\right)$ are the guiding-center coordinates with $\rho_{\|}=U / B$ and fields are evaluated at the guiding-center position $X$. This expression is correct to order $\epsilon_{B}$, and the 
presence of the vector $\mathbf{R}$ causes this one-form to be gyro-gauge invariant. (For more details see Refs. 7 and 8 and Appendix B.) We refer the reader to Appendix D for the expression of the Poisson brackets and unperturbed equations of motion using magnetic coordinates.

Consider now electromagnetic perturbations $(\delta \phi, \delta \mathrm{A})$ of the phase-space Lagrangian. In guiding-center coordinates the perturbed one-form is given as

$$
\gamma_{1}=\delta \mathbf{A}(\mathbf{X}+\rho) \cdot(d \mathbf{X}+d \rho)-\delta \phi(\mathbf{X}+\rho) d t
$$

where

$$
\rho=\rho_{\perp} \hat{\zeta}=\sqrt{\frac{2 \mu}{B}} \hat{\zeta}
$$

and

$$
d \rho=\frac{1}{\rho_{\perp} B} \hat{\zeta} d \mu+\rho_{\perp} \hat{\perp} d \zeta .
$$

We remind the reader that the tokamak configuration is treated as uniform when considering perturbations. Using extended phase space coordinates $\left(\mathbf{X}, \rho_{\|}, \mu, \zeta, t, w\right)$, the total phase-space Lagrangian is given as

$$
\gamma=\left(\mathbf{A}+\rho_{\|} \mathbf{B}-\mu \mathbf{W}+\epsilon \delta \mathbf{A}\right) \cdot d \mathbf{X}+\epsilon \frac{\partial \rho}{\partial \mu} \cdot \delta \mathbf{A} d \mu+\left(\mu+\epsilon \frac{\partial \rho}{\partial \zeta} \cdot \delta \mathbf{A}\right) d \zeta-H d t
$$

where

$$
H=\frac{1}{2} \rho_{\|}^{2} B^{2}+\mu B-w+\epsilon \delta \phi+\mathcal{O}\left(\epsilon_{B}^{2}\right)
$$

and

$$
\mathbf{W}=\mathbf{R}+\frac{1}{2}(\hat{b} \cdot \nabla \times \hat{b}) \hat{b}+O\left(\epsilon_{B}^{2}\right)
$$

and the parameters $\epsilon$ and $\epsilon_{B}$ define the gyrokinetic ordering and the tokamak ordering, respectively (see Appendix C).

\section{Gyrokinetic Formalisms}

Through the dependence of $(\delta \phi, \delta A)$ on $\rho$ (and therefore on $\zeta$ ), the symplectic structure and Hamiltonian acquire gyro-angle dependence. A gyrokinetic formalism represents a prescription for gyro-averaging the perturbed phase-space Lagrangian. Two generic $\mathbf{g y}^{-}$ rokinetic formalisms are possible.

The first formaljim is referred to as the gyrokinetic Hamiltonian formalism. It involves a transformation to gyro-center coordinates such that the phase-space Lagrangian becomes

$$
\bar{\gamma}=\left(\mathbf{A}+\overline{\boldsymbol{\rho}}_{\|} \mathbf{B}-\bar{\mu} \mathbf{W}\right) \cdot d \overline{\mathbf{X}}+\bar{\mu} d \bar{\zeta}-\bar{H}_{l} d t,
$$

where the Hamiltonian is given as

$$
\bar{H}_{I}=\frac{1}{2} \bar{\rho}_{\|}^{2} B^{2}+\bar{\mu} B-\bar{w}+\epsilon\left\langle\delta \Phi_{i}^{*}\right) .
$$


We note that the transformation has transferred the symplectic-structure perturbation to the Hamiltonian, and has produced a gyro-averaged effective perturbation potential $\left\langle\delta \Phi_{l}^{*}\right\rangle$, yet to be determined (see section III).

Since the symplectic structure is unchanged, we can still use the unperturbed Poisson brackets with the new gyro-center coordinates. The equations of motion are, therefore, given as

$$
\dot{\overline{\mathbf{X}}}=\frac{1}{B B_{\|}^{*}}\left(\mathbf{B} \times \bar{\nabla} \bar{H}_{I}+\mathbf{B}^{*} \frac{\partial \bar{H}_{I}}{\partial \bar{\rho}_{\|}}\right)
$$

and

$$
\dot{\bar{\rho}}_{\|}=-\frac{\mathbf{B}^{*}}{B B_{\|}^{*}} \cdot \bar{\nabla} \vec{H}_{I}
$$

where

$$
\mathbf{B}^{*}=\mathbf{B}+\bar{\rho}_{\|} \bar{\nabla} \times \mathbf{B}-\bar{\mu} \bar{\nabla} \times \mathbf{W}=B_{\|}^{*} \hat{\varepsilon}+\frac{\bar{\rho}_{\|}}{B^{2}} \mathbf{B} \times \bar{\nabla} \boldsymbol{P}+O\left(\epsilon_{B}^{2}\right),
$$

and $P$ is the equilibrium (unperturbed) jsotropic scalar pressure (divided by $4 \pi$ ) - we introduced the fluid-like quantity $\boldsymbol{P}$ by using the equilibrium pressure balance equation $\mathbf{J} \times \mathbf{B}=\nabla P$. This formalism is somewhat analogous to the $p_{\|}$-formulation of Hahm et al. ${ }^{12}$

The second formalism is referred to as the grrokinetic phase-space Lagrangian formalism. It involves a transformation to gyro-center coordinates such that the symplectic structure is left partially perturbed. The phase-space Lagrangian becomes

$$
\overline{\boldsymbol{\gamma}}=\left[\mathbf{A}+\bar{\rho}_{\|} \mathbf{B}-\bar{\mu} \mathbf{W}+\epsilon(\delta \mathbf{A})+\mathcal{O}\left(\epsilon^{2}\right)\right] \cdot d \overline{\mathbf{X}}+\tilde{\mu} d \bar{\zeta}-\bar{H}_{I I} d t
$$

where the Hamiltonian is given as

$$
\bar{H}_{I I}=\frac{1}{2} \bar{\rho}_{\|}^{2} B^{2}+\bar{\mu} B-\bar{w}+\epsilon\left\langle\delta \Phi_{H I}^{*}\right\rangle
$$

(This formalism is analogous to the $v_{\|}$-formulation contained in the Appendix of Hahm et al. ${ }^{12}$ ) Now that the symplectic structure has been perturbed, we obtain new expressions for the Poisson brackets. With these new brackets the equations of motion take the form

$$
\dot{\overline{\mathbf{X}}}=\frac{1}{B B_{\|}^{* *}}\left(\mathbf{B} \times \bar{\nabla} \bar{H}_{I I}+\mathbf{B}^{* *} \frac{\partial \bar{H}_{I I}}{\partial \bar{\rho}_{\|}}\right),
$$

and

$$
\dot{\bar{\rho}}_{\|}=-\frac{\mathbf{B}^{* *}}{B B_{\|}^{*}} \cdot\left[\bar{\nabla} \bar{H}_{I I}+\epsilon \frac{\partial(\delta \mathbf{A})}{\partial t}+O\left(\epsilon^{2}\right)\right]
$$

where

$$
\mathbf{B}^{* *}=\mathbf{B}^{*}+\epsilon\{\delta \mathbf{B}\rangle+\boldsymbol{O}\left(\epsilon^{2}\right)
$$


The presence of the inductive term of the perturbed electric field in the parallel equation of motion makes these gyro-center coordinates useful if one wishes to have this term appear explicitly. For our purposes, however, we shall use the gyrokinetic Hamiltonian formalism since its equations of motion appear in a simpler form. ${ }^{12}$ In any case, both formalisms describe the same physics using different gyro-center coordinates. (In particular, energy is conserved by the equations of motion in both cases.)

\section{GYROKINETIC HAMILTONLAN FORMALISM}

In this section we will show how to obtain the gyro-averaged phase-space lagrangian given by Eq. (5) through the use of the theory of Lie transformation of one-forms (as described briefly in Appendix A).

\section{A. Gyrokinetjc Hamiltonian}

1. Zeroth Order. The zeroth-order gyro-averaged term is functionally the same as the original one. Hence

$$
\bar{\gamma}_{0}=\left(\mathbf{A}+\bar{\rho}_{\|} \mathbf{B}-\bar{\mu} \mathbf{W}\right) \cdot d \overline{\mathbf{X}}+\bar{\mu} d \bar{\zeta}-\bar{H}_{0} d t
$$

and

$$
\tilde{H}_{0}=\frac{1}{2} \bar{\rho}_{\|}^{2} B^{2}+\bar{\mu} B-\bar{w} .
$$

2. First Order. The first-order gyro-averaged term is obtained from the expression

$$
\bar{\gamma}_{1}=\gamma_{1}-i_{1} \omega_{0}+d S_{1} .
$$

The terms on the right-hand side are identified, respectively, as the perturbation oneform $\gamma_{1}$, the inner product of the first-order generating vector field with the unperturbed Lagrange two-form, and the exterior derivative of the first-order phase-space gauge function.

The perturbation one-form is given by Eq. (1). The inner-praduct term is given by the expression

$$
i_{1} \omega_{0}=\left(\mathbf{B} \times \mathbf{G}_{1}+G_{1}^{\rho 1} \mathbf{B}\right) \cdot d \mathbf{X}-\mathbf{G}_{1} \cdot \mathbf{B} d_{\rho_{\|}}+G_{2}^{\mu} d \zeta-G_{1}^{\prime} d_{\mu}-\left(G_{1}^{\omega}+\rho_{\|} B^{2} G_{1}^{\rho^{\prime l}}+B G_{1}^{\mu}\right) d \mu,
$$

and the gauge-function term (which one is free to choose) is

$$
d S_{1}=\nabla S_{1} \cdot d \mathrm{X}+\frac{\partial S_{1}}{\partial \rho_{\|}} d \rho_{\|}+\frac{\partial S_{1}}{\partial \mu} d \mu+\frac{\partial S_{1}}{\partial \zeta} d \zeta
$$

where we have used the fact that time is not transformed through this process so that

$$
G_{1}^{*}=0=\frac{\partial S_{1}}{\partial w}
$$


The gyrokinetic Hamiltonian formalism requires that

$$
\bar{\gamma}_{1}=-\vec{H}_{1} d t
$$

so that the first-order generating vector field, determining the transformation from guidingcenter to gyro-center coordinates, is given by the following components

$$
\begin{aligned}
\overrightarrow{\mathbf{G}}_{1} & =-\frac{1}{B} \hat{b} \times\left(\delta \mathbf{A}+\bar{\nabla} S_{1}\right)-\frac{1}{B} \hat{b} \frac{\partial S_{1}}{\partial \vec{\rho}_{||}}, \\
\bar{G}_{1}^{\rho_{\|}} & =\frac{1}{B} \hat{b} \cdot\left(\delta \mathbf{A}+\bar{\nabla} S_{1}\right), \\
\bar{G}_{1}^{\mu} & =\frac{\partial \bar{\rho}}{\partial \bar{\zeta}} \cdot \delta \mathbf{A}+\frac{\partial S_{1}}{\partial \bar{\zeta}}, \\
\bar{G}_{1}^{\zeta} & =-\left(\frac{\partial \bar{\rho}}{\partial \bar{\mu}} \cdot \delta \mathbf{A}+\frac{\partial S_{1}}{\partial \bar{\mu}}\right), \\
-\bar{G}_{1}^{\omega} & =\frac{\partial S_{1}}{\partial t}+\bar{\rho}_{\mid} \mathbf{B} \cdot \bar{\nabla} S_{1}+B \frac{\partial S_{1}}{\partial \bar{\zeta}}-\delta \bar{\psi}_{1},
\end{aligned}
$$

and, consequently, the first-order Hamiltonian is given as

$$
\bar{H}_{1}=\langle\delta \phi\rangle-\bar{\rho}_{||} \mathbf{B} \cdot\langle\delta \mathbf{A}\rangle-\bar{\rho}_{\perp} B(\hat{\perp} \cdot \delta \mathbf{A}\rangle \doteq\langle\delta \phi\rangle-\langle\delta v\rangle=\left\langle\delta \psi_{1}\right\rangle .
$$

Note that the generating vector field components include terms related to the gauge function $S_{1}$. We will describe later on how to choose this function. We also note that this vector field is only needed if one wishes to go on to include higher-order terms. (For our purposes we need to go on to second order.)

3. Second Order. The second-order term is obtained from the expression

$$
\bar{\gamma}_{2}=0-i_{2} \omega_{0}-\frac{1}{2} i_{1}\left(\omega_{1}+\bar{\omega}_{1}\right)+d S_{2} .
$$

Since we wish to stop at this order, not all terms are relevant. Indeed, from the requirements of the gyrokinetic Hamiltonian formalism, we have

$$
\tilde{H}_{2}=\left(\left\langle i_{2}\right) \omega_{0}+\frac{1}{2}\left\langle i_{1} \omega_{1}\right\rangle+\frac{1}{2}\left\langle i_{1}\right\rangle \bar{\omega}_{1}\right)_{t} .
$$

The above expression indicates that we only need the gyro-averaged portion of the second order generating vector field. These are easily obtained and given as

$$
\left\langle\bar{G}_{2}^{\rho \|}\right\rangle=-\frac{1}{2 B} \hat{b} \cdot\left\langle\delta \mathbf{B} \times \overline{\mathbf{G}}_{1}+\left(\bar{G}_{1}^{\mu} \frac{\partial \delta \mathbf{A}}{\partial \bar{\mu}}+\bar{G}_{1}^{\zeta} \frac{\partial \delta \mathbf{A}}{\partial \bar{\zeta}}\right)\right\rangle,
$$

and

$$
\left\langle\bar{G}_{2}^{\mu}\right\rangle=-\frac{1}{2}\left\langle\bar{G}_{1}^{\mu}\left[\frac{\partial}{\partial \bar{\mu}}\left(\delta \mathbf{A} \cdot \frac{\partial \bar{\rho}}{\partial \bar{\zeta}}\right)-\frac{\partial}{\partial \bar{\zeta}}\left(\delta \mathbf{A} \cdot \frac{\partial \bar{\rho}}{\partial \bar{\mu}}\right)\right]-\overline{\mathbf{G}}_{1} \cdot\left(\frac{\partial \delta \mathbf{A}}{\partial \bar{\zeta}}-\bar{\nabla} \delta \mathbf{A} \cdot \frac{\partial \bar{\rho}}{\partial \bar{\zeta}}\right)\right\rangle
$$


The second-order Hamiltonian is given in terms of the first-order generating vector components and the perturbation potentials by the expression

$$
\begin{aligned}
\bar{H}_{2}= & \frac{1}{2} \bar{\rho}_{\|} \mathbf{B} \cdot\left\langle\delta \mathbf{B} \times \overline{\mathbf{G}}_{1}+\left(\bar{G}_{1}^{\mu} \frac{\partial}{\partial \bar{\mu}} \delta \mathbf{A}+\bar{G}_{i}^{\zeta} \frac{\partial}{\partial \bar{\zeta}} \delta \mathbf{A}\right)\right\rangle \\
& -\frac{1}{2} B\left\langle\overline{\mathbf{G}}_{1} \cdot\left(\frac{\partial}{\partial \bar{\zeta}} \delta \mathbf{A}-\bar{\nabla} \delta \mathbf{A} \cdot \frac{\partial \bar{\rho}}{\partial \bar{\zeta}}\right)-\bar{G}_{1}^{\mu}\left(\frac{\partial}{\partial \bar{\mu}}\left(\delta \mathbf{A} \cdot \frac{\partial \bar{\rho}}{\partial \bar{\zeta}}\right)-\frac{\partial}{\partial \bar{\zeta}}\left(\delta \mathbf{A} \cdot \frac{\partial \bar{\rho}}{\partial \bar{\mu}}\right)\right]\right\rangle \\
& -\frac{1}{2}\left\langle\overline{\mathbf{G}}_{1} \cdot\left(\bar{\nabla} \delta \phi+\frac{\partial}{\partial t} \delta \mathbf{A}\right)+\left(\bar{G}_{1}^{\mu} \frac{\partial}{\partial \bar{\mu}} \delta \phi+\bar{G}_{1}^{\phi} \frac{\partial}{\partial \bar{\zeta}} \delta \phi\right)\right\rangle \\
& -\frac{1}{2}\left(\left\langle\overline{\mathbf{G}}_{1}\right\rangle \cdot \bar{\nabla}\left(\delta \psi_{1}\right\rangle-\left\langle\bar{G}_{1}^{\rho^{\|}}\right\rangle \mathbf{B} \cdot\langle\delta \mathbf{A})+\left\langle\bar{G}_{1}^{\mu}\right\rangle \frac{\partial}{\partial \bar{\mu}}\left\langle\delta \psi_{1}\right\rangle\right) .
\end{aligned}
$$

4. Phase-space Gauge Function. We again emphasize that the choice of this gauge function is purely arbitrary. We make the particular choice for $S_{1}$ such that the "canonicalenergy" component of the first-order generating vector vanishes (i.e., $\bar{G}_{1}^{w}=0$ ). Making use of the gyrokinetic ordering, this means that to order $\varepsilon$ we have

$$
B \frac{\partial S_{1}}{\partial \bar{\zeta}}=\delta \bar{\psi}_{1}=\delta \bar{\phi}-\delta \bar{v},
$$

or

$$
S_{1} \doteq \frac{1}{B} \delta \bar{\Psi}_{1}=\frac{1}{B}(\delta \bar{\Phi}-\delta \tilde{\Upsilon}) .
$$

The second-order Hamiltonian, therefore, becomes

$$
\begin{aligned}
\tilde{H}_{2}= & \frac{1}{2}\left\langle(\hat{b} \cdot \delta \mathbf{A})^{2}\right\rangle-\frac{1}{2 \bar{B}}\left\langle\frac{\partial}{\partial \bar{\mu}}\left(\delta \bar{\psi}_{1}\right)^{2}+\frac{1}{B} \bar{\nabla} \delta \tilde{\Psi}_{1} \cdot \hat{b} \times \bar{\nabla} \delta \bar{\psi}_{1}\right) \\
& -\frac{1}{B}\left\langle\hat{b} \times \bar{\nabla} \delta \psi_{1} \cdot \delta \mathbf{A}\right\rangle+\frac{1}{2}\left\langle\frac{\partial}{\partial \bar{\zeta}} \delta \mathbf{A} \times \hat{b} \cdot \delta \mathbf{A}\right\rangle \\
& +B\left\langle\left(\delta \mathbf{A} \cdot \frac{\partial \bar{\rho}}{\partial \bar{\mu}}\right) \frac{\partial}{\partial \bar{\zeta}}\left(\delta \mathbf{A} \cdot \frac{\partial \bar{\rho}}{\partial \bar{\zeta}}\right)\right\rangle+\left\langle\left(\delta \mathbf{A} \cdot \frac{\partial \bar{p}}{\partial \bar{\mu}}\right) \frac{\partial}{\partial \bar{\zeta}^{\prime}} \delta \psi_{1}-\left(\delta \mathbf{A} \cdot \frac{\partial \bar{\rho}}{\partial \bar{\zeta}}\right) \frac{\partial}{\partial \bar{\mu}} \delta \psi_{1}\right)
\end{aligned}
$$

5. General Expression for $\delta \boldsymbol{A}$. The expression for the second-order Hamiltonian can be further simplified if we use two conditions imposed on the perturbed vector potential $\delta \mathrm{A}$. Consider the general expression for $\boldsymbol{\delta} \boldsymbol{A}$ as given by

$$
\delta \mathbf{A}=\alpha \mathbf{B}+\beta_{1} \nabla \psi_{\mathbf{p}}+\beta_{2} \hat{b} \times \nabla \psi_{p} .
$$

A first condition is the Coulomb gauge: $\nabla \cdot \delta \mathrm{A}=0$. The second condition stems from the observation that the perturbations under consideration have moderate to high $n$ values $(n \sim 5$ to 20). Combining those two conditions with the gyrokinetic and tokanak orderings, we obtain that $\beta_{2} \sim \beta_{1} / n$, and that $\delta B_{\|} \sim q n \beta_{1}+\beta_{2}$. From this we conclude that the 
relative contribution of the perturbation $\beta_{2}$ to $\delta B_{\|}$is $1 / q n^{2}$ smaller than that of the perturbation $\beta_{1}$ and, consequently, a useful representation for the perturbed vector potential is

$$
\delta \mathbf{A}=\alpha \mathbf{B}+\beta \nabla \psi_{p} .
$$

Using $\hat{\mathbf{1}}=\nabla \psi_{\mathbf{p}} /\left|\nabla \psi_{\mathbf{p}}\right|=\hat{\mathbf{2}} \times \hat{b}$, and the particle velocity unit vectors

$$
\hat{\perp}=-(\sin \zeta \hat{1}+\cos \zeta \hat{2})=\hat{\zeta} \times \hat{b},
$$

the expression for the first-order effective perturbed potential is given as

$$
\delta \psi_{1}=\delta \phi-\bar{\rho}_{||} B^{2} \alpha+\bar{\rho}_{\perp} B\left|\bar{\nabla} \psi_{p}\right| \beta \sin \bar{\zeta} .
$$

Finally, the second-order Hamiltonian is given by the expression (keeping terms of order $\epsilon^{2}$ only)

$$
\begin{aligned}
\vec{H}_{2}= & \frac{1}{2}\left\langle(\bar{b} \cdot \delta \mathbf{A})^{2}\right\rangle+B\left\langle\left(\delta \mathbf{A} \cdot \frac{\partial \bar{\rho}}{\partial \bar{\mu}}\right) \frac{\partial}{\partial \bar{\zeta}}\left(\delta \mathbf{A} \cdot \frac{\partial \bar{\rho}}{\partial \bar{\zeta}}\right)\right\rangle \\
& -\frac{1}{B}\left\langle\hat{b} \times \bar{\nabla} \delta \psi_{1} \cdot \delta \mathbf{A}\right\rangle-\frac{1}{2 B}\left\langle\frac{\partial}{\partial \bar{\mu}}\left(\delta \bar{\psi}_{1}\right)^{2}+\frac{1}{B} \bar{\nabla} \delta \bar{\Psi}_{1} \cdot \bar{b} \times \bar{\nabla} \delta \bar{\psi}_{1}\right\rangle \\
& +\left\langle\left(\delta \mathbf{A} \cdot \frac{\partial \bar{\rho}}{\partial \bar{\mu}}\right) \frac{\partial}{\partial \bar{\zeta}} \delta \psi_{1}-\left(\delta \mathbf{A} \cdot \frac{\partial \bar{\rho}}{\partial \bar{\zeta}}\right) \frac{\partial}{\partial \bar{\mu}} \delta \psi_{1}\right\rangle .
\end{aligned}
$$

From the linear (perturbation) term $(\boldsymbol{V} \cdot \delta \mathbf{A}\rangle$, we recognize the nonlinear term $\left\langle\delta v_{E} \cdot \delta \mathbf{A}\right\rangle$ where the effective perturbed $\delta \mathbf{E} \times \mathbf{B}$ velocity is given as

$$
\delta \mathbf{v}_{E}=-\frac{1}{B} \bar{\nabla} \delta \psi_{1} \times \hat{b}
$$

The other terms are simply ponderomotive potentials related to the transformation from guiding-center coordinates to gyro-center coordinates.

\section{B. Gyrokinetic Vlasov Equation}

1. Gyrokinetic Hamiltonian. The gyrokinetic Hamiltonian is given by the expression

$$
\bar{H}=\frac{1}{2} \bar{\rho}_{\|}^{2} B^{2}+\bar{\mu} B-\bar{w}+\epsilon\left\langle\delta \Phi^{*}\right\rangle,
$$

where the effective perturbed potential is given as

$$
\left\langle\delta \Phi^{*}\right\rangle=\langle\delta \phi\rangle-\bar{\rho}_{\|} \mathbf{B} \cdot\langle\delta \mathbf{A}\rangle-\bar{\rho}_{\perp} \boldsymbol{B}\langle\hat{\perp} \cdot \delta \mathbf{A}\rangle+\mathcal{O}(\epsilon) .
$$

2. Gyro-center Coordinates. The guiding-center to gyro-center phase space transformation is expressed as

$$
Z^{a}=\bar{Z}^{\mathrm{a}}-\epsilon \bar{G}_{1}^{a}+\mathcal{O}\left(\epsilon^{2}\right)
$$


More specifically, we have $w=\bar{w}$, and

$$
\begin{aligned}
\mathbf{X} & =\overline{\mathbf{X}}+\frac{\epsilon}{B}\left[\hat{b} \times\left(\delta \mathbf{A}+\frac{1}{B} \bar{\nabla} \delta \bar{\Psi}_{1}\right)+\frac{\hat{b}}{B} \frac{\partial}{\partial \overline{\boldsymbol{\rho}}_{\|}} \delta \bar{\Psi}_{1}\right], \\
\rho_{\|} & =\overline{\boldsymbol{\rho}}_{\|}-\frac{\epsilon}{B} \hat{b} \cdot \delta \mathbf{A}, \\
\mu & =\bar{\mu}-\epsilon\left(\delta \mathbf{A} \cdot \frac{\partial \bar{\rho}}{\partial \bar{\zeta}}+\frac{1}{B} \delta \bar{\psi}_{1}\right), \\
\zeta & =\bar{\zeta}+\epsilon\left(\delta \mathbf{A} \cdot \frac{\partial \bar{\zeta}}{\partial \bar{\mu}}+\frac{1}{B} \frac{\partial}{\partial \bar{\mu}} \delta \bar{\Psi}_{1}\right) .
\end{aligned}
$$

3. Gyrokinetic Vlasov Equation. The gyrokinetic Vlasov equation is given by the expression

$$
\frac{\partial}{\partial t} \bar{F}+\left(\bar{V} \hat{b}+\dot{\bar{X}}_{\perp}\right) \cdot \bar{\nabla} \bar{F}+\dot{\bar{\rho}}_{\|} \frac{\partial}{\partial \bar{\rho}_{\|}} \bar{F}=0,
$$

where the parellel velocity is

$$
\bar{V}=\hat{b} \cdot \dot{\bar{X}}=\bar{\rho}_{\|} B+\epsilon \frac{1}{B} \frac{\partial}{\partial \bar{\rho}_{\|}}\left\langle\delta \Phi^{*}\right\rangle=\bar{\rho}_{\|} B-\epsilon \bar{b} \cdot\langle\delta \mathbf{A}\rangle+\mathcal{O}\left(\epsilon^{2}\right),
$$

the perpendicular velocity is

$$
\dot{\mathbf{X}}_{\perp}=\frac{B}{B_{\|}^{*}}\left[\mathbf{V}_{D}+\epsilon \frac{1}{B} \frac{\partial}{\partial \bar{\rho}_{\|}}\left\langle\delta \Phi^{*}\right\rangle \bar{\rho}_{\|} \frac{\hat{b}}{B^{2}} \times \bar{\nabla} P+\epsilon \frac{\dot{b}}{B} \times \bar{\nabla}\left\langle\delta \Phi^{*}\right\rangle\right],
$$

where $V_{D}$ is the unperturbed magnetic drift velocity

$$
\mathrm{V}_{D}=\frac{\hat{b}}{B} \times\left(\bar{\mu} \bar{\nabla} B+\bar{\rho}_{\|}^{2} B^{2} \hat{b} \cdot \bar{\nabla} \hat{b}\right)=\left(\bar{\mu}+\bar{\rho}_{\|}^{2} B\right) \frac{\hat{b}}{B} \times \bar{\nabla} B+\bar{\rho}_{\|}^{2} \frac{\hat{b}}{B} \times \bar{\nabla} P
$$

and the parallel equation of motion is

$$
\dot{\bar{\rho}}_{\|}=-\frac{1}{B B_{\|}^{*}} \mathbf{B}^{*} \cdot\left[\left(\bar{\mu}+\bar{\rho}_{\|}^{2} B\right) \bar{\nabla} B+\epsilon \bar{\nabla}\left(\delta \Phi^{*}\right)\right]
$$

In magnetic coordinates, the expressions for the drift velocities are ${ }^{16}$

$$
\begin{aligned}
\dot{\psi}_{p} & =-\frac{g}{D}\left(\mu+\rho_{\|}^{2} B\right) \frac{\partial B}{\partial \theta}+\frac{\epsilon}{D}\left(I \frac{\partial}{\partial \xi}\left\langle\delta \Phi^{*}\right)-g \frac{\partial}{\partial \theta}\left\langle\delta \Phi^{*}\right)\right) \\
\dot{\theta} & =\frac{V}{D}\left[B_{\|}^{*}+B g \rho_{\|}\left(\frac{P^{\prime}}{B^{2}}\right)\right]+\frac{g}{D}\left(\mu+\rho_{\|}^{2} B\right) \frac{\partial B}{\partial \psi_{p}}+\frac{\epsilon}{D}\left(g \frac{\partial}{\partial \psi_{p}}\left\langle\delta \Phi^{*}\right\rangle-\delta \frac{\partial}{\partial \xi}\left\langle\delta \Phi^{*}\right)\right) \\
\dot{\xi} & =\frac{V}{D}\left[q B_{\|}^{*}-B I \rho_{\|}\left(\frac{P^{\prime}}{B^{2}}\right)\right]+\frac{1}{D}\left(\mu+\rho_{\|}^{2} B\right)\left(\delta \frac{\partial B}{\partial \theta}-I \frac{\partial B}{\partial \psi_{p}}\right)+\frac{\epsilon}{D}\left(\delta \frac{\partial}{\partial \theta}\left\langle\delta \Phi^{*}\right)-1 \frac{\partial}{\partial \psi_{p}}\left\langle\delta \Phi^{*}\right)\right) .
\end{aligned}
$$


Similarly for the parallel equation of motion

$$
\begin{aligned}
\dot{\rho}_{\|}= & -\frac{1}{D}\left[\frac{B_{\|}^{*}}{B}+g \rho_{\|}\left(\frac{P^{\prime}}{B^{2}}\right)\right]\left(\mu+\rho_{\|\|}^{\tilde{\varepsilon}} B\right) \frac{\partial B}{\partial \theta} \\
& -\frac{\epsilon}{D}\left\{\left[\frac{B_{\|}^{*}}{B}+g \rho_{\|}\left(\frac{\rho^{\prime}}{B^{2}}\right)\right] \frac{\partial}{\partial \theta}\left\langle\delta \Phi^{*}\right\rangle+\left[q \frac{B_{\|}^{*}}{B}-I \rho_{\|}\left(\frac{P^{\prime}}{B^{2}}\right)\right] \frac{\partial}{\partial \xi}\left\langle\delta \Phi^{*}\right)\right\},
\end{aligned}
$$

and finally the toroidal momentum equation is

$$
\dot{P}_{\xi}=-\varepsilon \frac{\partial}{\partial \xi}\left\langle\delta \Phi^{*}\right\rangle,
$$

where the $\xi$-axis is the axisymmetry direction, and the overbar notation was ignored in the previous expressions.

\section{GYROKINATIC MAXWELL'S EQUATIONS}

The set of Maxweli's equations relate perturbation potentials $(\delta \phi, \delta \mathrm{A})$ to density (current) integrals involving the total distribution function alone (Poisson's equation) or with some componert of the particle velocity (Ampere's equation). Together with the gyrokinetic energy integral invariant and the gyrokinetic Vlasov equation, they represent a selfconsistent and energy-conserving set of nonlinear equations suitable for purposes of gyrokinetic particle simulation.

We will now express those density integrals in terms of the gyro-center distribution function $\bar{F}$ and gyro-center coordinates $\left(\overline{\mathbf{X}}, \overline{\rho_{1}}, \bar{\mu}, \bar{\zeta}\right)$. A brief description of this procedure is outlined in Appendix A.

\section{A. Guiding-Center Transformation}

The density integral for the Poisson equation involves the zeroth velocity moment. It transformation under the operation $\left(T_{G C}^{*}\right)^{-1}$ is trivial and we get $\left(T_{G C}^{*}\right)^{-1} 1=1$. The density integral for the Ampere equation involves the first velocity moment. Its transformation under the operation $\left(T_{G c}^{*}\right)^{-1}$ gives

$$
\left(T_{G C}^{*}\right)^{-1} \mathbf{v} \doteq \mathrm{V}_{G C}=U \hat{b}+\mathrm{V}_{D}+\left(\rho_{\perp} B \hat{\perp}+\dot{V}_{D}\right),
$$

where the expression for $V_{G C}$ is derived in Appendix B. Here we note that the gyroaverage of $\mathbf{V}_{G C}$ gives the unperturbed guiding-center drift velocity, i.e., $U$ is the parallel drift velocity while $V_{D}$ is the magnetic drift velocity; the vectors $\hat{L}$ and $\hat{V}_{D}$ are purely oscillatory.

\section{B. Gyrokinetic Maxwell's Equations}

From the expressions of the density integrals in terms of the guiding-center distribution and guiding-center coordinates, we now obtain the expressions in terms of the gyro-center 
quantities. Using the expression for $\mathbf{V}_{G C}$, the gyrokinetic Maxwell's equations are given as

$$
\begin{aligned}
\nabla^{2} \delta \phi & =-4 \pi \sum_{a}\left(\frac{e}{m}\right)_{a} \int \Omega_{a} B_{\|}^{*} d^{8} \overline{\mathbf{Z}}\left(T_{G Y}^{*} \vec{F}_{a}\right)(\overline{\mathbf{Z}}) \delta^{3}\left(T_{G C}^{*} \overline{\mathbf{X}}-\mathbf{x}\right), \\
\nabla^{2} \delta \mathbf{A} & =\frac{4 \pi}{c}\left[\mathbf{J}-\sum_{a}\left(\frac{e}{m}\right)_{a} \int \Omega_{a} B_{\|}^{*} d^{6} \overline{\mathbf{Z}} \mathbf{V}_{G C}(\overline{\mathbf{Z}})\left(T_{G Y}^{*} \bar{F}_{a}\right)(\overline{\mathbf{Z}}) \delta^{3}\left(T_{G C}^{*} \overline{\mathbf{X}}-\mathbf{x}\right)\right],
\end{aligned}
$$

where $\mathbf{J}$ is the equilibrium (unperturbed) current, and $T_{G Y}^{*} \bar{F}=\bar{F}-\epsilon \frac{\hat{b}}{B} \times\left(\delta \mathbf{A}+\frac{1}{B} \bar{\nabla}_{\delta} \bar{\Psi}_{1}\right) \cdot \bar{\nabla} \bar{F}+\epsilon \frac{\hat{b}}{B} \cdot \delta \mathbf{A} \frac{\partial}{\partial \bar{\rho}_{\|}} \bar{F}+\epsilon\left(\delta \mathbf{A} \cdot \frac{\partial \bar{\rho}}{\partial \bar{\zeta}}+\frac{1}{B} \delta \bar{\psi}_{1}\right) \frac{\partial}{\partial \bar{\mu}} \bar{F}+\cdots$, and $T_{G C}^{*} \overline{\mathbf{X}}=\overline{\mathbf{X}}+\bar{\rho}_{\perp} \hat{\zeta}+\cdots$. These equations are correct to order $\varepsilon^{2}$ and $\varepsilon_{B}$.

\section{Gyrokinetic Energy Integral Invariant}

We will now derive an expression for the gyrolinetic energy integral invariant using the procedure outlined in Appendix A. The simplest way to proceed is to transform the particle kinetic energy integral into the guiding-center kinetic energy integral and then transform it into the gyro-center kinetic energy integral.

The guiding-center kinetic energy integral is given by the expression

$$
E_{\text {oh }}=\int \Omega B_{\|}^{*}\left[\frac{1}{2} \rho_{\|}^{2} B^{2}+\mu B+O\left(\varepsilon_{B}^{2}\right)\right] E(Z) d^{0} \mathbf{Z},
$$

and the gyro-center kinetic energy integral is given as

$$
E_{G k}=j \Omega B_{\|}^{*}\left\langle\left[\left(T_{G Y}^{*}\right)^{-1}\left(\frac{1}{2} \bar{\rho}_{\|\}}^{2} B^{2}+\bar{\mu} B\right)\right]\right\rangle \bar{F}(\overline{\mathbf{Z}}) d^{\infty} \overline{\mathrm{Z}},
$$

where $d^{5} \overline{\mathbf{Z}}=2 \pi d^{3} \overline{\mathbf{X}} d \bar{\rho} \| d \bar{\mu}$, and

$$
\begin{aligned}
& \left\langle\left[\left(T_{G Y}^{*}\right)^{-1}\left(\frac{1}{2} \bar{\rho}_{\|}^{2} B^{2}+\bar{\mu} B\right)\right]\right\rangle= \\
& \frac{1}{2} \bar{\rho}_{\| l}^{2} B^{2}+\bar{\mu} B-\bar{\rho}_{\|} B \hat{b} \cdot\{\delta \mathbf{A}\rangle-\bar{\rho}_{\perp} B\langle\delta \mathbf{A} \cdot \hat{\perp}\rangle+\frac{1}{2}\left\langle(\delta \mathbf{A} \cdot \hat{b})^{2}\right\rangle+\frac{1}{B}\left\langle\delta \mathbf{A} \cdot \hat{b} \times\left[\bar{\rho}_{\|} \mathbf{B} \times \delta \mathbf{B}\right]\right\rangle \\
& +\frac{1}{2 B}\left\langle\frac{\partial}{\partial \bar{\mu}}\left[(\delta \bar{\phi})^{2}-(\delta \bar{v})^{2}\right]\right\rangle+\frac{1}{2 B^{2}}\langle\bar{\nabla} \delta \bar{\Phi} \cdot \hat{b} \times \bar{\nabla} \delta \bar{\phi}-\bar{\nabla} \delta \tilde{\Upsilon} \cdot \hat{b} \times \bar{\nabla} \delta \tilde{v}\rangle \\
& +B\left\langle\left(\delta \mathbf{A} \cdot \frac{\partial \bar{\rho}}{\partial \bar{\zeta}}\right) \frac{\partial}{\partial \bar{\zeta}}\left(\delta \mathbf{A} \cdot \frac{\partial \bar{\rho}}{\partial \bar{\mu}}\right)\right\rangle+\left\langle\left(\delta \mathbf{A} \cdot \frac{\partial \bar{\rho}}{\partial \bar{\zeta}}\right) \frac{\partial}{\partial \bar{\mu}} \delta v-\left(\delta \mathbf{A} \cdot \frac{\partial \bar{\rho}}{\partial \bar{\mu}}\right) \frac{\partial}{\partial \bar{\zeta}} \delta v\right\rangle .
\end{aligned}
$$

The total gyrokinetic energy integral can finally be given by the expression

$$
\begin{aligned}
E_{G t}= & \sum_{a} \int \Omega_{a} B_{\|}\left\langle\left(\left(T_{G Y}^{*}\right)^{-1}\left(\frac{1}{2} \bar{\rho}_{\|}^{2} B^{2}+\bar{\mu} B\right)\right]\right\rangle_{a} \bar{F}_{a} d^{\delta} \overline{\mathbf{Z}} \\
& +\frac{1}{8 \pi} \int\left(|\delta \mathbf{E}|^{2}+|\mathbf{B}+\delta \mathbf{B}|^{2}\right) d^{3} \mathbf{x} .
\end{aligned}
$$




\section{DISCUSSION AND CONCLUSIONS}

We have succeeded in deriving self-consistent, energy-conserving, nonlinear gyrokinetic Maxwell-Vlasov equations suitable for gyrokinetic particle simulation studies of finite $\beta$ tokarnak microturbulence.

This work reproduces and generalizes the results of all previous derivations of sets of equations suitable for gyrokinetic particle simulation by treating fully electromagnetic perturbations in tokamal geometry. Because the magnetic-coordinate representation of the gyro-center equations of motion [Eqs. (25) and (26)] preserved the simple form of the guiding-center equations of White and Chance, ${ }^{\text {ts }}$ these equations can also be used non-selfconsistently to study the diffusion of test particles in a given turbulent tokamak plasma, or a tokamak with partially destroyed magnetic surfaces. ${ }^{25}$

We have presented the gyrokinetic Hamiltonian formalism and used it to derive a nonlinear set of equations. This formalism was only one of the many formalisms obtainable from the Action-variational Lie perturbation method. Such versatility is quite useful in numerical studies since we can adapt a formalism to a particular numerical scheme. All such formalisms involve the introduction of phase-space gauge scalar fields, in addition to generating vector fields, that facilitate the gyro-averaging and simplification of various quantities.

The presence of the fluid-like quantity $\hat{b} \times \nabla P$ in the expressions for $B^{*}$ and $V_{D}$ was justified as follows. The unperturbed magnetic configuration used in our work was that of an equilibrium tolcamak configuration with prescribed plasma current and pressure profiles and magnetic configuration. These unperturbed fields are related through the equilibrinm pressure balance equation $\mathbf{J} \times \mathbf{B}=\nabla P$. Such an equation was used to show explicitly the equilibrium-pressure effects in the expression for the gyro-center drift velocity [e.g. Eq. (23)].

Future work related to this nonlinear grrokinetic set of equations will include the derivation of the moment equations obtzined from the gyrohinetic Vlasov equation [Eq. (21)], and their comparison with the various RMHD theories and their FLR-kinetic modifications. (See for instance Hategarra and Wakatan ${ }^{17}$ and Hazeltine et al. ${ }^{18}$ ) A comparison with the generalized gyrokinetics of Bernstein and Catto ${ }^{20}$ will also be made.

\section{ACKNOWLEDGMENTS}

I am grateful for useful discussions with Drs. R.B. White, L. Chen, T.S. Hahm, J.A. Krommes, W.W. Lee, and W.M. Tang. This work was supported by the U.S. Department of Energy under contract DE-AC02-76-CHO-3073. 


\section{Appendix A. Theory of Lie Transformation}

The basic references for this appendix are Dragt and Finn, ${ }^{19}$ Littlejohn, ${ }^{13}$ and Cary and Littlejohn. ${ }^{14}$

\section{Notation and Terminology}

Consider the transformation of a point $Z$ by the operator $T$. The new point $\bar{Z}$ is related to the old point $Z$ through the relation $\bar{Z}=T Z$. The transformation operator $T$ induces a transformation on scalar fields, called pull-back and noted $T^{*}$, with the scalar-invariance property $F(Z)=\bar{F}(\bar{Z})$ or $F=T^{*} \bar{F}$. If we consider the more general case of differential forms (the scalar fields are considered as zero-forms), the pull-back of forms can be represented as $\bar{\gamma}_{k}=\left(T^{*}\right)^{-1} \gamma_{k}$, where $\gamma_{k}$ and $\bar{\gamma}_{k}$ are $k$-forms, i.e., they are k-covariant antisymmetric tensors. Finally, vector fields $\mathbf{V}$ are transformed by the push-forward operator $T$. induced by the transformation operator $T$, and represented as $\overline{\mathrm{V}}=T . \mathrm{V}$.

The theory of Lie transformation defines the transformation $T^{\mathbf{e}}$ as a continuous transformation (with respect to the parameter $\epsilon$ ) generated by the vector field $G$, such that

$$
T^{*}=\exp (\epsilon G) \text {. }
$$

The expressions for the pull-back and push-forward operators are given in terms of the Lie derivative $L_{G}$ with respect to the vector field $G$ as

$$
T^{* *}=\exp \left(\epsilon L_{G}\right)=\left(T_{*}^{*}\right)^{-1}
$$

\section{Iterative Sequences of Tie Transformations}

Our analysis uses Lie transformation theory to gyro-average various quantities. Those quantities are normally given as series expansions in terms of the parameter $\epsilon_{\text {. It is, }}$ therefore, customary to proceed through a sequence of transformations $T^{*}=\cdots T_{3}^{*} T_{2}^{*} T_{1}^{*}$, where the transformation $T_{n}^{*}$ removes the gyro-angle dependence of the $n$-th order term of the serjes expansion.

\section{Transformation of a One-form}

The fundamental one-form in Hamiltonian dynamics is the Poincaré-Cartan one-form $\gamma=\mathbf{p} \cdot d \mathbf{q}-H d t$ (given in terms of canonical coordinates). In a way similar to the use of potentials $(\psi, A)$ in electromagnetics, it comes into play only through its exterior derivative $d \gamma$. When the one-form $\gamma$ is transformed, a phase-space gauge function $S$ is normally added to produce a transformed one-form with some desired properties. Hence for our purposes one-forms are transformed according to the expression

$$
\vec{\gamma}=\left(T^{e *}\right)^{-1} \gamma+d S \text {. }
$$

Using the series expansions for $\gamma, \bar{\gamma}$, and $S$

$$
(\gamma, \vec{\gamma}, S)=\sum_{n=0}^{\infty} \epsilon^{n}\left(\gamma_{n}, \bar{\gamma}_{n}, S_{n}\right),
$$


(where $S_{0}$ is normally taken to be zero) and the inverse pull-back sequence

$$
\left(T^{e *}\right)^{-1}=\cdots\left(T_{2}^{\varepsilon *}\right)^{-1}\left(T_{1}^{\epsilon *}\right)^{-1}=I d-\epsilon L_{1}+\epsilon^{2}\left(\frac{1}{2} L_{1}^{2}-L_{2}\right)+\cdots,
$$

we get the following expressions (order by order)

$$
\begin{aligned}
& \bar{\gamma}_{0}=\gamma_{0}, \\
& \bar{\gamma}_{1}=\gamma_{1}-L_{1} \gamma_{0}+d S_{1}, \\
& \bar{\gamma}_{2}=\gamma_{2}-L_{1} \gamma_{1}+\left(\frac{1}{2} L_{1}^{2}-L_{2}\right) \gamma_{0}+d S_{2},
\end{aligned}
$$

We can simplify these expressions by using the homotopy formula $L_{X}=i_{X} d+d i_{X}$, where $i_{X}$ is the inner product with the vector field $X$ to produce

$$
\begin{aligned}
& \bar{\gamma}_{0}=\gamma_{0}, \\
& \bar{\gamma}_{1}=\gamma_{1}-i_{1} \omega_{0}+d S_{1}^{\prime}, \\
& \bar{\gamma}_{2}=\gamma_{2}-i_{2} \omega_{0}-\frac{1}{2} i_{1}\left(\omega_{1}+\bar{\omega}_{1}\right)+d S_{2}^{\prime},
\end{aligned}
$$

where $\omega_{n}=d \gamma_{n}, i_{n} \omega_{m}=G_{n}^{a}\left(\omega_{m}\right)_{a b} d Z^{b}$, and $S_{1}^{\prime}=S_{1}-d i_{1} \gamma_{0}$, etc.

\section{Transformation of a Density Integral}

The Maxwell's equations relate, on the one hand, a potential $\chi$ acted on by some spacetime differential operator $L$ and, on the other hand, a density integral involving the particle distribution function $f$ and some component of the particle velocity. A representative equation can be given as

$$
\begin{aligned}
L \chi\left(x^{\prime}\right) & =\int d^{3} v g(z) f(z) \\
& =\int d^{2} z g(z) f(x) \delta^{3}\left(x-x^{\prime}\right),
\end{aligned}
$$

where $\mathbf{z}=(\mathbf{x}, \boldsymbol{v})$ and $d^{6} \mathbf{z}=d^{3} \mathbf{x} d^{x} \mathbf{v}$. The transformation from physical-space particle coordinates $\mathbf{z}$ to guiding-center coordinates $Z=T_{G C} \mathbf{z}$ produces the expression

$$
L_{\chi}\left(\mathbf{x}^{\prime}\right)=\int B B_{\|}^{*} d^{d} \mathbf{Z} F(\mathbf{Z})\left[\left(T_{G C}^{*}\right)^{-1} g\right](\mathbf{Z}) \delta^{3}\left(T_{G C}^{-1} \mathbf{X}-\mathbf{x}^{\prime}\right)
$$

In the integral expression given above, the variable $\mathbf{Z}$ is a dummy variable. We, therefore, substitute the gyro-center coordinates $\bar{Z}=T_{G Y} \mathbf{Z}$, and then use the relation $F=T_{G Y}^{*} \bar{F}$ to produce

$$
L_{\chi}\left(\mathbf{x}^{\prime}\right)=\int B B_{\|}^{*} d^{\beta} \overline{\mathbf{Z}}\left[T_{G Y}^{*} \bar{F}\right](\overline{\mathbf{Z}})\left[\left(T_{G C}^{*}\right)^{-1} g\right](\overline{\mathbf{Z}}) \delta^{3}\left(T_{G C}^{-1} \overline{\mathbf{X}}-\mathbf{x}^{\prime}\right)
$$


This procedure can be similarly applied to the particle kinetic energy integral which involves the integral

$$
\int d^{6} \mathbf{z} g(\mathbf{z}) f(\mathbf{z})
$$

and can, therefore, be transformed into

$$
\int B B_{i \|}^{*} d^{8} \overline{\mathbf{Z}} \bar{F}(\overline{\mathbf{Z}})\left\langle\left(T_{G Y}^{*}\right)^{-1}\left[\left(T_{G C}^{*}\right)^{-1} g\right](\overline{\mathbf{Z}})\right\rangle
$$

\section{Appendix B. Gyro-gauge Invariant Phase-space Lagrangian}

In this appendix, we present a moditied version of Littlejohn's work on guiding-center theory, ${ }^{7}$ and present a critical discussion of the work of Hagan and Frieman. ${ }^{11}$

\section{Gyro-gauge Invariance Formalism}

We begin with the expression for the phase-space Lagrangian given in terms of the noncanonical coordinates $\left(x^{\prime}, u, \mu_{0}, \theta\right)$

$$
\gamma=\frac{1}{\epsilon}\left[A+\epsilon\left(u \hat{b}+\sqrt{2 \mu_{0} B} \hat{\perp}\right)\right] \cdot d x^{\prime}-\left(\frac{1}{2} u^{2}+\mu_{0} B\right) d t
$$

where $\varepsilon$ is the ratio of the particle gyroradius to some characteristic nonuniformity length scale (the $\epsilon$-scaling shown above is typical of guiding-center theory), and the unperturbed magnetic field is assumed time-independent.

Instead of proceeding as in Ref. 7 (i.e., by using a two-step transformation: preparatory - guiding-center position - and gyro-averaging), we ransform directly to the guidingcenter coordinates $(\mathbf{X}, U, \mu, \zeta)$ which must satisfy the following Lagrange-bracket conditions (compare with Ref. 11).

$$
[\mu, \zeta]=\epsilon,[\zeta, Z]=0, Z \neq \mu .
$$

These conditions effectively place all geometrically dependent terms into the new gyroangle $\zeta$ (as was also accomplished in Ref. II - see the end of this appendix for a discussion of their approach), and indicate that the phase-space Lagrangian has been gyro-averaged.

Following the procedure presented in Ref. 13 and outlined in Appendix A, we obtain the guiding-center phase-space Lagrangian 


$$
\begin{aligned}
& \gamma_{G}=\frac{1}{\epsilon}\left\{\mathbf{A}+\epsilon U \hat{b}-\epsilon^{2}\left[\mu \mathbf{R}+\dot{b}\left(G_{1}^{U}+U \sqrt{\frac{2 \mu}{B}} \hat{b} \cdot \nabla \hat{b} \cdot \dot{\zeta}+\mu \hat{\zeta} \cdot \nabla \hat{b} \cdot \dot{\perp}\right)\right.\right. \\
& \left.\left.+\mathrm{B} \times \mathrm{G}_{2}-\sqrt{\frac{\mu B}{2}} G_{10}^{\zeta} \hat{\zeta}+\left(U \sqrt{\frac{2 \mu}{B}}(\dot{b} \cdot \nabla \times \dot{b})+\frac{B}{2 \sqrt{2 \mu B}} G_{1}^{\mu}-\frac{\mu}{2 B} \dot{\zeta} \cdot \nabla B\right) \dot{L}\right]\right\} \cdot d \mathbf{X} \\
& \left.+\epsilon ; \mu-\epsilon\left(\frac{\mu U}{B}(\hat{b} \cdot \nabla \times \hat{b})+G_{1}^{\mu}-\frac{\mu}{3 B} \sqrt{\frac{2 \mu}{B}} \bar{\zeta} \cdot \nabla B-\frac{\partial}{\partial \zeta} S_{3}\right)\right] d \zeta \\
& +\epsilon^{2}\left(\frac{\partial}{\partial \mu} S_{3}+G_{10}^{\alpha}\right) d \mu+\epsilon^{2}\left[G_{2} \cdot \dot{b}+\frac{\partial}{\partial U} S_{3}\right] d U \\
& -\left[\frac{1}{2} U^{2}+\mu B-\epsilon\left(U G_{1}^{U}+B G_{1}^{\mu}-\mu \sqrt{\frac{2 \mu}{B}} \hat{\zeta} \cdot \nabla B\right)\right] d t
\end{aligned}
$$

where the spatial component of the first-order generating vector $\mathbf{G}_{1}=-\sqrt{\frac{2 \mu}{B}} \bar{\zeta}$ was used. The other generating vector components and the phase-space gauge function $S_{3}$ will be chosen so as to gyro-average the phase-space Lagrangian $\gamma_{G}$, as well as give it a simple form. In accordance with Littlejohn, ${ }^{7,8,13,24}$ the term $-\epsilon \mu R \cdot d X$ must be kept to ensure gyro-gauge invariance (to order $\epsilon^{2}$ ), and we have $G_{1}^{\zeta}=G_{10}^{\zeta}+\mathbf{G}_{1} \cdot \mathbf{R}$.

We now proceed with the gyro-averaging of the one-form $\gamma_{G}$. Without going into the details of this operation, we simply give the result as

$$
\begin{aligned}
\gamma_{G}= & \frac{1}{\epsilon}\left\{\mathbf{A}+\epsilon U \hat{b}-\epsilon^{2}\left[\mu \mathbf{R}+\left(\left\langle G_{1}^{U}\right)-\frac{\mu}{2} \hat{b} \cdot \nabla \times \hat{b}\right) \hat{b}\right]\right\} \cdot d \mathbf{X} \\
& +\epsilon\left[\mu-\epsilon\left(\left(G_{1}^{\mu}\right\rangle+\frac{\mu U}{B} \dot{b} \cdot \nabla \times \hat{b}\right)\right] d \zeta \\
& -\left[\frac{1}{2} U^{2}+\mu B-\epsilon\left(U\left\langle G_{1}^{U}\right\rangle+B\left\langle G_{1}^{\mu}\right)\right)\right] d t .
\end{aligned}
$$

At this point, various possibilitiea leading to a simplification of $\gamma_{G}$ exist. For example, Littlejohn ${ }^{7,8}$ chooses

$$
\left\langle G_{1}^{U}\right\rangle=\frac{\mu}{2} \dot{b} \cdot \nabla \times \hat{b}
$$

and

$$
\left\langle G_{1}^{\mu}\right\rangle=-\frac{\mu U}{B} \dot{b} \cdot \nabla \times \hat{b}
$$

so that

$$
\gamma_{G}=\frac{1}{\epsilon}\left(\mathbf{A}+\epsilon U \hat{b}-\epsilon^{2} \mu \mathbf{R}\right) \cdot d \mathbf{X}+\epsilon \mu d \zeta-\left(\frac{1}{2} U^{2}+\mu B+\frac{\epsilon}{2} \mu U \hat{b} \cdot \nabla \times \hat{b}\right) d t .
$$


In a later paper, ${ }^{24}$ Littlejohn decides on the more favorable choice

$$
\left\langle G_{1}^{U}\right\rangle=\mu \hat{b} \cdot \nabla \times \hat{b}
$$

and

$$
\left\langle G_{1}^{\mu}\right\rangle=-\frac{\mu U}{B} \hat{b} \cdot \nabla \times \hat{b},
$$

so that

$$
\gamma_{G}=\frac{1}{\epsilon}\left[\mathbf{A}+\epsilon U \dot{b}-\epsilon^{2} \mu\left(\mathbf{R}+\frac{1}{2}(\hat{b} \cdot \nabla \times \hat{b}) \dot{b}\right)\right] \cdot d \mathbf{X}+\epsilon \mu d \zeta-\left(\frac{1}{2} U^{2}+\mu B\right) d t,
$$

which is also the choice made by Hagan and Frieman. ${ }^{11}$ This choice allows us to transform to coordinates in which the expression for the parallel drift velocity $U=u+\mu_{0} \hat{b} \cdot \nabla \times \hat{b}+\cdots$ contains the Baños term explicitly ${ }^{21}$ and, therefore, gives a simple form to the guiding-center Hamiltonian. Following the discussion in Ref. 9 on the difference between the guidingcenter formalism and the gyrokinetic formalism, we set $\epsilon=1$ in the last expression for $\gamma_{G}$ and obtain Eq. (1).

\section{Guiding-center Transformation of Particle Velocity}

With this last choice, the components of the first-order generating vector are

$$
\begin{aligned}
& G_{1 g c}=-\sqrt{\frac{2 \mu}{B}} \hat{\zeta}, \\
& G_{1 g c}^{U}=\mu \hat{b} \cdot \nabla \times \hat{b}-\frac{\mu}{2}[\hat{\zeta} \cdot \nabla \hat{b} \cdot \hat{\perp}+\hat{\perp} \cdot \nabla \hat{b} \cdot \hat{\zeta}]-U \sqrt{\frac{2 \mu}{B}} \hat{b} \cdot \nabla \hat{b} \cdot \dot{\zeta} \\
& G_{1 g c}^{\mu}=-\frac{U}{B} G_{1 g c}^{U}+\frac{\mu}{B} \sqrt{\frac{2 \mu}{B}} \hat{\zeta} \cdot \nabla B \\
& G_{1 g c}^{\zeta}=\mathbf{G}_{1 p c} \cdot \mathbf{R}+\sqrt{\frac{2 \mu}{B}} \frac{\hat{\perp}}{B} \cdot \nabla B+\frac{U^{2}}{B \sqrt{2 \mu B}} \hat{b} \cdot \nabla \hat{b} \cdot \hat{\perp}-\frac{U}{4 B}[\hat{\zeta} \cdot \nabla \hat{b} \cdot \hat{\zeta}-\hat{\perp} \cdot \nabla \dot{b} \cdot \hat{\perp}]_{+}
\end{aligned}
$$

where $\mathbf{R}=\nabla \hat{\perp} \cdot \hat{\zeta}=\nabla \hat{1} \cdot \hat{2}$ and $\hat{\perp}=-(\sin \zeta \hat{1}+\cos \zeta \hat{2})$, and the expression for $\mathbf{G}_{\mathbf{z}_{\rho c}}$ is not needed for our purposes.

In section IV the expression for the transformation of the particle velocity vector $\mathbf{V}_{G C}=$ $\left(T_{G C}^{*}\right)^{-1} v$ was needed. Up to order $\epsilon_{B}$, this expression is given as

$$
\begin{aligned}
\mathbf{V}_{G C}-\mathbf{v} & =-\left(G_{1 \rho c} \cdot \nabla \mathbf{v}+G_{1 \rho c}^{U} \hat{b}+\frac{B}{\sqrt{2 \mu B}} G_{1 g c}^{\mu} \hat{\perp}-\sqrt{2 \mu B} G_{1_{g c}}^{\zeta}\right) \\
& =\mathbf{V}_{D}+\dot{V}_{D},
\end{aligned}
$$

where the gyro-averaged part is the magnetic drift velocity

$$
\mathrm{V}_{D}=\frac{\hat{b}}{B} \times\left(\mu \nabla B+U^{2} \hat{b} \cdot \nabla \hat{b}\right),
$$


and the purely oscillatory part is

$$
\begin{aligned}
\overline{\mathrm{V}}_{D}= & -\left[G_{1 g c}^{U}+2 \mu \hat{\zeta} \cdot \nabla \dot{b} \cdot \dot{\perp}\right] \hat{b} \\
& +\left[\frac{1}{2} \sqrt{\frac{2 \mu}{B}} U\left(\hat{\zeta} \cdot \nabla \hat{b} \cdot \bar{\perp}+\frac{1}{2} \hat{b} \cdot \nabla \times \hat{b}\right)+\frac{\mu}{B} \dot{\zeta} \cdot \nabla B\right] \hat{\perp} \\
& +\left[\frac{1}{4} \sqrt{\frac{2 \mu}{B}} U(\hat{\perp} \cdot \nabla \hat{b} \cdot \bar{\perp}+3 \hat{\zeta} \cdot \nabla \tilde{b} \cdot \hat{\zeta})+\frac{\mu}{B} \dot{\perp} \cdot \nabla B\right] \hat{\zeta}
\end{aligned}
$$

\section{Comment on a paper of Hagan and Frieman}

Finally, we would like to comment on the work of Hagan and Frieman. ${ }^{11}$ They correctly point out that the initial Darboux-Lie transformation approach of Littlejohn, ${ }^{A, 7}$ as applied by Yang and Choi, ${ }^{10}$ leads to gyro-averaged equations that depend on the orientation of the unit vectors perpendicular to the magretic field. This dependence is not present in the results of the standard method (i.e., as in Ref. 5) and, therefore, they look for a new method which will avoid such an undesirable feature.

Before proceeding however, they criticize the gyro-gauge invariance formalism of Littlejohn ${ }^{7}$ in which the introduction of a scalar field $\psi(x)$, which is involved in the transformation of the gyto-angle $\zeta \longmapsto \zeta+\psi$, is chosen such as to eliminate the geometrically dependent terms from the gyro-averaged equations. Their criticism of the formalism is based on the observation that this elimination leads to a magnetic differential equation for $\psi$ that does not satisfy the integrability condition of Newcomb. ${ }^{22}$

The integrability condition 22 says that the magnetic differential equation $\hat{b} \cdot \nabla \psi=S$ where $S$ is a given scalar function, has a single-valued solution $\psi$ only if $S$ satisfies the condition

$$
\oint S \frac{d \ell}{B}=0
$$

where the line integral is taken around a closed field line.

By requiring that the gyro-averaged equations be gyro-gauge independent, Hagan and Frieman $^{11}$ obtain

$$
\hat{b} \cdot \nabla \psi=\frac{1}{2} \hat{b} \cdot \nabla \times \hat{b}-\hat{b} \cdot \mathbf{R} \doteq S,
$$

where $\mathbf{R}=\nabla \hat{\mathbf{i}} \cdot \hat{\mathbf{2}}$. They point out that this equation clearly violates the integrability condition, and conclude that the gyro-gauge invariance formalism leads to an "ill-behaved" $\psi$ (our emphasis). We claim that this condition should not be imposed on the gyro-gauge field $\psi$ (requiring it to be single-valued) because this field is angle-like in nature and, therefore, multivalued. Hence, no inconsistency should be associated with the gyro-gauge formalism.

Hagan and Frieman ${ }^{1 "}$ then go on to develop a new method based on a transformation 
to coordinates which satisfy the Poisson-bracket conditjons

$$
\{\mu, \zeta\}=-\frac{1}{\epsilon},\{\mu, Z\}=0, Z \neq \zeta
$$

Because of the simple relation between Poisson brackets and Lagrange brackets (Appendix D), we recognize the conditions we have used above. We have thus established the relationship between our work and the work of Hagan and Frieman. ${ }^{11}$ Since the gyro-gauge formalism does not lead to any inconsistency, they are equivalent methods.

\section{Appendix C. Gyrokinetic and Tokamak Orderings}

\section{Gyrokinetic Ordering}

We use the nonlinear gyrokinetic ordering introduced by Frieman and Chen. ${ }^{5}$ The ratjo of perturbed quantities over equilibrium quantities is assumed to be of order $\epsilon \ll 1$ (defined as $\rho_{i} / L_{n}$ where $\rho_{i}$ is the ion gyro-radius and $L_{n}$ is the length scale associated with the equilibrium density gradient - normally the perpendicular equilibrium length $L_{0 \perp}$ (minor radius)], i.e.,

$$
\left|\frac{\delta F}{F}\right| \sim\left|\frac{\delta B}{B}\right| \sim O(\epsilon)
$$

and we also have

$$
\left|\frac{e \delta \phi}{T}\right| \sim\left|\frac{\delta V_{E}}{V_{t}}\right| \sim\left|\frac{\delta P}{P}\right| \sim O(\epsilon),
$$

where $V_{t}$ is some characteristic (thermal) velocity. In addition, the derivative of the perturbed quantities are assumed to scale as follows

$$
\left|\frac{\partial}{\Omega \partial t}\right| \sim\left|\rho_{\hat{i}} \hat{b} \cdot \nabla\right| \sim \mathcal{O}(\epsilon)
$$

and

$$
\left|\rho_{i} \hat{b} \times \nabla\right| \sim \mathcal{O}(1)
$$

\section{Tokamak Ordering}

a. Equilibrium Magnetic Field. Using strajght-field-line magnetic coordinates $\left(\psi_{p}, \theta, \xi\right)$, the covariant representation for the axisymmetric tokamak magnetic field is ${ }^{16,23}$

$$
\mathbf{B}=g\left(\psi_{p}\right) \nabla \xi+I\left(\psi_{p}\right) \nabla \theta+\delta\left(\psi_{p}, \theta\right) \nabla \psi_{p}
$$

where $\mathbf{B} \cdot \nabla \psi_{p}=0$, and the scalar pressure $P\left(\psi_{p}, \theta\right)$ is self-consistently associated with the functions $g, I$, and $\delta$. (For circular unshifted magnetic surfaces: $\delta=0 .{ }^{18}$ ) The Jacobian of the transformation from physical-space coordinates to magnetic coordinates is given as

$$
J^{-1}=\nabla \psi_{p} \times \nabla \theta \cdot \nabla \xi=\frac{B^{2}}{(g q+I)}
$$




\section{b. Tokamak Ordering.}

We associate a length scale $L_{B}$ to the equilibrium magnetic field nonuniformity. For the tokamak geometry this length turns out to be the parallel equilibrium length $L_{0 \| \mid}$ (major radius). We define a second small parameter $\epsilon_{B}$ where

$$
\epsilon_{B}=\rho_{i} / L_{B}=\epsilon \epsilon_{0} \ll 1,
$$

with $\varepsilon_{0}=L_{n} / L_{B}=L_{0 \perp} / L_{0 \|}$ being the inverse aspect ratio. For small-inverse-aspect-ratio tokamak geometries we have $\epsilon_{B} \ll \epsilon$.

The equilibrium tokamak configuration for low- $\beta$ plasmas $\left(P / B^{2} \sim \epsilon_{0}^{2}\right)$ scales as follows

$$
\left|\frac{\rho_{i}}{B} \hat{b} \times \nabla B\right| \sim\left|\rho_{i} \hat{b} \cdot \nabla \hat{b}\right| \sim\left|\rho_{i} \hat{b} \cdot \nabla \times \hat{b}\right| \sim O\left(\epsilon_{B}\right),
$$

and

$$
\left|\frac{\rho_{i}}{B} \hat{b} \cdot \nabla B\right| \sim O\left(\epsilon_{B} \epsilon_{0}\right)
$$

so that

$$
\left|\frac{V_{D}}{V_{t}}\right| \sim\left|\frac{\bar{V}_{D}}{V_{t}}\right| \sim\left|\frac{B_{\|}^{*}-B}{B}\right| \sim\left|\rho_{i} \mathbf{R}\right| \sim O\left(\epsilon_{B}\right) .
$$

\section{Appendix D. Poisson Brackets and Equations of Motion}

\section{Gyro-gveraged Phase-space Lagrangian}

The gyro-gauge-independent phase-space Lagrangian derived in Appendix B and used in section II is given as

$$
\gamma=\left(\mathbf{A}+\rho_{\|} \mathbf{B}-\mu \mathrm{W}\right) \cdot d \mathbf{X}+\mu d \zeta-\left(\frac{1}{2} \rho_{\|}^{2} B^{2}+\mu B\right) d t,
$$

and is correct to order $\epsilon_{B}$. Using the covariant representation for the equilibrium magnetic field, given in Appendix C, with its associated vector potential

$$
\mathbf{A}=\psi \nabla \theta-\psi, \nabla \xi,
$$

we obtain the magnetic-coordinate representation for the phase-space Lagrangian ${ }^{24}$

$$
\gamma=\psi d \theta-\psi_{p} d \xi+\rho_{\|}\left(g d \xi+I d \theta+\delta d \psi_{p}\right)+\mu d \zeta-H_{0} d t
$$




\section{Lagrange and Poisson Brackets}

The Lagrange brackets are obtained from the components of the two-form $\omega=d \gamma$ as follows ${ }^{13}$

$$
\omega_{i j}=\left[z^{i}, z^{j}\right]=\frac{\partial}{\partial z^{\mathbf{i}}} \gamma_{j}-\frac{\partial}{\partial z^{j}} \gamma_{i} .
$$

There is a simple relationship between Lagrange brackets and Poisson brackets. From the Lagrange covariant tensor we create a matrix whose coefficients are the components of the Lagrange tensor, i.e., the Lagrange brackets. Inverting this matrix (assuming it is nonsingular), we obtain a matrix whose coefficients are the Poisson brackets. The determinant of the Lagrange matrix is $D^{2}$ where

$$
D=(g q+I)+\rho_{\|}\left[g\left(I^{\prime}-\partial_{\theta} \delta\right)-g^{\prime} I\right] .
$$

A coordinate-free, gyro-gauge-independent representation for the Poisson bracket is given as

$$
\begin{aligned}
\{F, G\}= & \left(\frac{\partial}{\partial \zeta} F \frac{\partial}{\partial \mu} G-\frac{\partial}{\partial \mu} F \frac{\partial}{\partial \zeta} G\right) \\
& -\frac{1}{B B_{\|}^{*}} \mathbf{B} \cdot\left[\left(\nabla F+\mathbf{W} \frac{\partial}{\partial \zeta} F\right) \times\left(\nabla G+\mathbf{W} \frac{\partial}{\partial \zeta} G\right)\right] \\
& +\frac{1}{B B_{\|}^{*}} \mathbf{B}^{*} \cdot\left[\left(\nabla F+\mathbf{W} \frac{\partial}{\partial \zeta} F\right) \frac{\partial}{\partial \rho_{\|}} G-\left(\nabla G+\mathbf{W} \frac{\partial}{\partial \zeta} G\right) \frac{\partial}{\partial \rho_{i \|}} F\right]
\end{aligned}
$$

where

$$
\mathbf{B}^{*}=\mathbf{B}+\rho_{\|} \nabla \times \mathbf{B}+\mathcal{O}\left(\epsilon_{B}^{2}\right)=B_{\|}^{*} \hat{b}+\rho_{\|}(\nabla \times \mathbf{B})_{\perp} \text {, }
$$

and

$$
B_{\|}^{*}=\dot{b} \cdot \mathbf{B}^{*}=\frac{D}{J B} .
$$

\section{Unperturbed Equations of Motion}

Using the expression for the unperturbed guiding-center Hamiltonian

$$
H_{0}=\frac{1}{2} \rho_{\|}^{2} B^{2}+\mu B,
$$

and the expression for the Poisson bracket given above, we get the following equations of motion.

a. Guiding-center Drifts

$$
\begin{aligned}
\dot{\mathbf{X}} & =\left\{\mathbf{X}, H_{0}\right\} \\
& =\frac{1}{B B_{\|}^{*}}\left(\mathbf{B} \times \nabla H_{0}+\mathbf{B}^{*} B U\right),
\end{aligned}
$$


where $U=\rho_{\|} B$ is the parallel drift velocity. Using magnetic coordinates we find the following expressions (correct to order $\left.\epsilon_{B}\right)^{16}$

$$
\begin{aligned}
\dot{\psi}_{p} & =-\frac{g}{D}\left(\mu+\rho_{\|}^{2} B\right) \frac{\partial}{\partial \theta} B \\
\dot{\theta} & =\frac{g}{D}\left(\mu+\rho_{\|}^{2} B\right) \frac{\partial}{\partial \psi_{p}} B+\frac{U}{D}\left(B_{\|}^{*}+\rho_{\|} g B \frac{P^{\prime}}{B^{2}}\right) \\
\dot{\xi} & =\frac{1}{D}\left(\mu+\rho_{\|}^{2} B\right)\left(\delta \frac{\partial}{\partial \theta} B-I \frac{\partial}{\partial \psi_{p}} B\right)+\frac{U}{D}\left(q B_{\|}^{*}-I \rho_{\|} B \frac{P^{\prime}}{B^{2}}\right)
\end{aligned}
$$

b. Parallel Equation of Motion

$$
\begin{aligned}
\dot{\rho}_{\|} & =\left\{\rho_{\|,}, H_{0}\right\} \\
& =-\frac{1}{B B_{\|}^{*}} \mathbf{B}^{*} \cdot \nabla H_{0} \\
& =-\frac{1}{D}\left(1-\rho_{\|} g^{\prime}\right)\left(\mu+\rho_{\|}^{2} B\right) \frac{\partial}{\partial \theta} B .
\end{aligned}
$$

c. Toroidal Momentum Equation

From the expression for the gyro-averaged phase-space Lagrangian

$$
\gamma=-\left(\psi_{p}-\rho_{\|} g\right) d \xi+\cdots,
$$

we obtain an expression for the gyro-averaged toroidal momentum $P_{\ell}=-\left(\psi_{p}-P_{\|} g\right)$, and we can easily verify that it satisfies the toroidal momentum equation

$$
\dot{P}_{\xi}=\left\{P_{\xi}, H_{0}\right\}=0 .
$$




\section{References}

${ }^{1}$ W.W. Lee, Phys. Fluids 26, 556 (1983).

${ }^{2}$ W.W. Lee, J. Comp. Phys. 72, 243 (1987).

${ }^{3}$ W.W. Lee, J.A. Krommes, C. Oberman, and R.A. Smith, Phys. Fluids 27, 2652 (1984).

${ }^{4}$ W.W. Lee and W.M. Tang, Phys. Fluids 31, 612 (1988).

${ }^{5}$ E.A. Frieman and L. Chen, Phys. Fluids 25, 502 (1982).

${ }^{8}$ R.G. Littlejohn, J. Math. Phys. 20, 2445 (1979).

7R.G. Littlejohn, Phys. F'luids 24, 1730 (1981).

${ }^{8}$ R.G. Littlejohn, J. Plasma Phys. 28, 111 (1983).

${ }^{9}$ D.H.E. Dubin, J.A. Krommes, C. Oberman, and W.W. Lee, Phys. Fluids 26, 3524 (1983).

${ }^{10}$ S-C. Yang and D-I. Choi, Phys. Lett. A 108, 25 (1985).

${ }^{11}$ W.K. Hagan and E.A. Frieman, Phys. Fluids 28, 2641 (1985).

${ }^{12}$ T.S. Hahm, W.W. Lee, and A. Brizard, Phys. Fluids 31, I940 (1988).

${ }^{13}$ R.G. Littlejohn, J. Math. Phys. 23, 742 (1982).

14J.R. Cary and R.G. Littlejohn, Ann. Phys. 151, 1 (1983).

${ }^{15}$ T.S. Hahm, Princeton Plasma Physics Laboratory Report No. PPPL-2513 (May, 1988).

To appear in Phys. Fluids (Sept. 1988).

${ }^{16}$ R.B. White and M.S. Chanve, Phys. Fluids 27, 2455 (1984).

${ }^{17}$ A. Hasegawa and M. Wabatani, Phys. Fluids 26, 2770 (1983).

${ }^{18}$ R.D. Hazeltine, C.T. Hsu, and P.H. Morrison, Phys. Fluids 30, 3204 (1987).

${ }^{19}$ A.J. Dragt and J.M. Finn, J. Math. Phys. 17, 2215 (1976).

${ }^{20}$ R.G. Littlejohn, Physica Scripta T2/1, 119 (1982).

${ }^{21}$ T.G. Northrop and J.A. Rome, Phys. Fluids 21, 384 (1978).

${ }^{22}$ W.A. Newcomb, Phys. Fluids 2, 362 (1959).

${ }^{23}$ R.B. White, A.H. Boozer, and R. Hay, Phys. Fluids 25, 575 (1982). 
${ }^{24}$ R.G. Littlejohn, Phys. Fluids 28, 2015 (1985).

${ }^{25}$ A.H. Boozer and R.B. White, Phys. Rev. Lett. 49, 786 (1982).

${ }^{28}$ I.B. Bernstein and P.J. Catto, Phys. Fluids 28, 1342 (1985). 